\title{
Pérdida de la audición neurosensorial en el adulto: Revisión
}

de tema.

Neurosensory hearing loss in the adult: literature review.

Daniela Umaña Conejo' Daniela Montero Madrigal. ${ }^{2}$ Geremy Rodríguez Villalobos ${ }^{3}$

1,2 Médico general. Trabajador independiente, San José Costa Rica. 3 Médico general Caja costarricense de seguro social, Alajuela Costa Rica.

Contacto: danielaumac@gmail.com danymma@gmail.com geremy10@gmail.com

\section{RESUMEN}

El Estudio de Carga Global de Enfermedad del año 2015 determinó que la pérdida de audición es la cuarta causa de discapacidad globalmente, afectando a 6-8\% de la población mundial. La pérdida de audición periférica se clasifica en conductiva, neurosensorial o mixta. Su principal efecto es la afectación de la comunicación y el principal factor de riesgo en el caso de pérdida auditiva neurosensorial es la edad. Dentro de las causas de pérdida de la audición se encuentran procesos degenerativos relacionados con la edad, mutaciones genéticas, exposición a ruidos altos o medicamentos ototóxicos y condiciones crónicas. Su inicio es insidioso y debido a la estigmatización de la enfermedad, el diagnóstico usualmente es tardío. La rehabilitación de la persona con pérdida de audición debe manejarse de forma integral; sumado al uso de aparatos auditivos, debe asegurarse el bienestar psicológico del paciente y su compatibilidad con el dispositivo para de ese modo, mejorar su calidad de vida.

Palabras Clave: Pérdida de la audición, oído, discapacidad.

\section{ABSTRACT}

Cómo citar:

Conejo, D. U., Montero

Madrigal , D. ., \&

Rodríguez Villalobos,

G. PÉRDIDA DE

LA AUDICIÓN

EN EL ADULTO:

REVISIÓN DEL TEMA.

Revista Ciencia $Y$

Salud Integrando Conocimientos, 5(4),

Pág. 47-52. https://

doi.org/10.34192/

cienciaysalud.

v5i4.300

Recibido: 19/mar/2021

Aceptado: 22/ago/2021

Publicado: 04/sep/2021
The 2015 Global Burden of Disease Study determined that hearing loss is the fourth leading cause of disability globally, affecting $6-8 \%$ of the world's population. Peripheral hearing loss is classified as conductive, sensorineural or mixed. Its main effect is impaired communication and the main risk factor for neurosensory hearing loss is age. Among the most important causes of hearing loss are agerelated degenerative processes, genetic mutations, exposure to loud noises or ototoxic medications and chronic conditions. Its onset is insidious and due to the stigmatization of the disease, the diagnosis is usually late. Rehabilitation of the patient with hearing loss must be managed holistically; in addition to the use of hearing aids, it must ensure the psychological well-being of the patient and its compatibility with the device, thereby improving quality of life.

Keywords: EHearing loss, ear, impairment 


\section{MATERIALES Y MÉTODOS}

La presente revisión está fundamentada a partir de una búsqueda de información actualizada en distintas fuentes bibliográficas, específicamente: PubMed, SCIELO, ScienceDirect y ClinicalKey. Se llevó a cabo una recopilación de información relacionada a pérdida de la audición en el adulto y su anatomía, fisiología, etiología, factores de riesgo, diagnóstico, tratamiento, rehabilitación y prevención. Se consideraron libros y artículos científicos de revisión y de estudios experimentales publicados entre 2017-2020.

\section{INTRODUCCIÓN}

La pérdida de audición es un trastorno común cuyos efectos pueden variar desde un grado casi indetectable de discapacidad hasta una alteración profunda en la capacidad de funcionar en la sociedad (1). Para el entendimiento de sus consecuencias debe considerarse la edad de inicio, la severidad, la velocidad de la pérdida y cualquier audición residual (2). Su inicio es insidioso y debido a que se acompaña de estrategias compensatorias, es comúnmente pasado por alto por el médico y el paciente. La pérdida de audición periférica se clasifica en conductiva (causada por alteración en el oído externo o medio), neurosensorial (producto de disfunción en cóclea o ganglio espiral) o mixta (3). El principal efecto de la pérdida de audición es la afectación de la comunicación y si no se trata, puede afectar indirectamente la salud, el ámbito psicosocial y económico; llevando a aislamiento social y reducción de la calidad de vida. Incluso, algunos efectos psicológicos de la pérdida de audición incluyen paranoia, depresión, irritabilidad y ansiedad (2).

\section{ANATOMÍA Y FISIOLOGÍA}

El sistema auditivo periférico se divide en tres partes: oído externo (formado por oreja y canal auditivo externo), medio (contiene los huesecillos auditivos y limita hacia lateral con membrana timpánica y hacia medial con la cóclea) e interno (órgano encargado de audición y equilibrio formado por los canales semicirculares, el vestíbulo y la cóclea) (2).

El canal auditivo externo permite la transmisión de las ondas de sonido a la membrana timpánica, y ésta por medio de los huesecillos auditivos, genera vibración acoplando la energía del sonido proveniente del canal externo con la cóclea llena de endolinfa en el oído interno (4). El movimiento en ondas del fluido endolinfático distorsiona las células ciliadas del órgano de Corti para convertir la fuerza mecánica en una señal electroquímica que se propaga por el nervio auditivo y es interpretada como sonido finalmente (2).

\section{EPIDEMIOLOGÍA}

El Estudio de Carga Global de Enfermedad del año 2015 midió los años vividos con discapacidad y determinó que la pérdida de audición es la cuarta causa de discapacidad global, afectando a 6-8\% de la población mundial $(5,6)$. Su estudió mostró que la prevalencia es mayor en hombres y niños que en mujeres y niñas; además, indicó que la prevalencia aumenta bruscamente entre los 20-64 años de edad (5). Otros estudios demuestran que la prevalencia de pérdida de la audición en niños y adultos es mayor en países de pequeños y medianos ingresos que en naciones de ingresos altos $(6,7)$.

\section{FACTORES DE RIESGO}

La edad es el principal factor de riesgo para la pérdida de la audición neurosensorial. Hay evidencia de que la audición empieza a deteriorarse en la tercera década; sin embargo, no es clínicamente evidente hasta la quinta o sexta década (8). La exposición prolongada o continua a ruidos altos (recreacional y ocupacional) y medicamentos ototóxicos como aminoglucósidos, salicilatos, furosemida y agentes quimioterapéuticos deben considerarse pues son factores importantes $(7,8)$. 


\section{CIENCIA\&SALDD}

\section{ETIOLOGÍA}

La pérdida de audición es causada por alteración o daño a cualquier porción del sistema auditivo central o periférico (3). La pérdida auditiva conductiva resulta de la obstrucción o enfermedad del oído externo o medio que evita la transmisión de la energía del sonido al oído interno. El tratamiento médico o quirúrgico usualmente restaura completamente la audición (2). Dentro de las principales causas de pérdida de la audición neurosensorial se encuentran procesos degenerativos relacionados con la edad, mutaciones genéticas, exposición a ruidos altos o medicamentos ototóxicos y condiciones crónicas (3).

- Edad avanzada: es la causa más común. Generalmente es bilateral, simétrica y más pronunciada a altas frecuencias. Además, se caracteriza por la reducción en la capacidad de entender el habla aún con sonidos suficientemente altos (1, 3).

- Mutaciones genéticas: la incidencia de la pérdida de audición severa a profunda difiere entre países desarrollados y no desarrollados; y se encuentra influenciada por causas genéticas y ambientales (6).

- Exposición a ruidos altos: puede ser exposición ocupacional o recreacional. La falta de prevención y consciencia de las consecuencias de exposición excesiva aumenta el riesgo (6). El ruido daña las células ciliadas en el oído interno por estrés mecánico directo de intensa presión del sonido y al activar vías moleculares inducidas por estrés, incluyendo la generación de especies reactivas de oxígeno y sobrecarga de calcio $(3,9)$.

- Exposición a medicamentos ototóxicos: los principales medicamentos con efectos adversos son los aminoglucósidos y cisplatino; ambos son tóxicos para las células ciliadas sensoriales (3). En muchos de estos medicamentos, los beneficios son mayores que los riesgos de pérdida de la audición; por ejemplo la gentamicina en la sepsis neonatal. El peligro aumenta cuando no se tiene precaución y no se monitorea la audición (6).

- Otoesclerosis: es una de las causas más comunes de hipoacusia progresiva en adultos jóvenes entre la segunda y cuarta década de vida y que en la mayoría de casos es bilateral. Se caracteriza por la presencia de tejido óseo muy denso, menos vascularizado y por ende más compacto (esclerótico). En la otoscopia se describe el signo de Schwartze, que indica la presencia de zonas hiperémicas en el promontorio que son visibles a través de la membrana timpánica sana y traduce otoesclerosis activa. La valoración clínica y una audiometría usualmente son suficientes para hacer el diagnóstico (10).

- Enfermedad de Meniere: condición idiopática del laberinto membranoso en la que la cantidad de endolinfa en la escala media es excesiva y se caracteriza por episodios espontáneos de vértigo, pérdida de la audición fluctuante y tinitus. Afecta principalmente a adultos entre 30 y 60 años (11). El tratamiento puede ser médico o quirúrgico e incluye el uso de antihistamínicos, benzodiacepinas, anticolinérgicos, cambios dietarios (limitar sal, cafeína y alcohol) y hasta cirugía del saco endolinfático (12).

- Nutrición: literatura reciente de estudios epidemiológicos y en animales demuestra que la deficiencia de ciertos micronutrientes y manifestaciones agudas y crónicas de malnutrición proteica están asociadas a pérdida de la audición; por ejemplo deficiencia de vitamina A (6).

- Inmune: la prevalencia de condiciones que lleven a pérdida de audición como lupus, colitis ulcerativa y artritis reumatoide no es clara, pero se cree que son responsable de menos de $1 \%$ de todos los casos de alteración de la audición (6).

\section{DIAGNÓSTICO}

El tamizaje de pérdida de audición se puede realizar por medio de cuestionarios auto-administrados, pruebas como el frote de dedos, voz susurrada y la audiometría, ya que son las pruebas más precisas y fáciles de realizar; no obstante, no se cuenta con suficiente evidencia para evaluar el balance entre los beneficios y los 
daños del tamizaje de pérdida de audición en adultos asintomáticos mayores de 50 años (13).

El paciente con discapacidad auditiva se presenta con reconocimiento propio de alteración en su audición o con preocupación por parte de familiares que han notado la alteración. En la historia clínica se debe preguntar al paciente la duración, lateralidad, fluctuación y progresión de la pérdida de audición; así como antecedentes patológicos, historia de trauma de cabeza u oído, exposición a medicamentos e historia familiar de condiciones auditivas. A partir del examen físico es importante evaluar el oído por impactación de cerumen, anormalidades del canal auditivo externo, perforación de membrana timpánica, supuración. Debe incluirse valoración de pares craneales, cabeza y cuello $(8,13)$.

Aquellos pacientes en quienes se sospeche pérdida de la audición, deben ser referidos para una audiometría. La prueba se realiza en cabinas aisladas de sonido y demuestra la capacidad del paciente de detectar sonido presentado a diferentes frecuencias (8).

\section{TRATAMIENTO}

El médico debe proveer información sobre la naturaleza y causas de la pérdida de audición, así como dispositivos de ayuda (si aplica) y protección auditiva. En caso de historia de medicamentos ototóxicos, se debe suspender el medicamento (13).

La Administración de Medicamentos y Alimentos define los audífonos como un dispositivo amplificador de sonido que busca compensar la discapacidad auditiva. Su objetivo es mejorar la percepción de conversaciones bajas o música y otros sonidos mientras se asegura que los sonidos no sean ruidos incómodos $(3,13)$.

El implante coclear es un dispositivo colocado mediante cirugía que evita porciones dañadas del oído y estimula directamente el nervio auditivo. Se utiliza en pacientes con pérdida auditiva neurosensorial bilateral moderada-profunda que no se benefician con el uso de audífonos (13). El implante permite la restauración parcial de la audición y ha mostrado mejora en la percepción del sonido, el funcionamiento social y psicológico y la calidad de vida del paciente (3).

En la actualidad, se estudia el potencial para activar células progenitoras en la cóclea para la regeneración de células ciliadas junto con terapias basadas en células para repoblar la población de neuronas del ganglio espiral o administrar neurotrofinas para la protección de las neuronas del ganglio espiral después de la pérdida auditiva (4).

\section{REHABILITACIÓN}

La rehabilitación de la persona con pérdida de audición debe manejarse de forma integral; sumado al uso de aparatos auditivos debe asegurarse el bienestar psicológico del paciente y su compatibilidad con el dispositivo. Para ello, es importante la educación del paciente respecto a síntomas de pérdida de audición para la detección temprana, el apoyo psicológico y social que le permita adaptarse al dispositivo y la promoción de actitudes y comportamientos positivos (14). La rehabilitación ha demostrado disminuir el aislamiento social, la depresión y el deterioro cognitivo (4). Además, alienta a los pacientes con pérdida de la audición a usar tácticas de escucha y estrategias de comunicación que mejoren el uso de señales visuales para facilitar la recepción del sonido y consecuentemente el acceso a conversaciones del día a día (15).

\section{PREVENCIÓN}

Muchas personas con pérdida de audición tratan de disimularlo porque es usualmente asociado a la edad y a baja inteligencia. El estigma puede impedir el tratamiento temprano y además disminuir la autoestima y autoeficacia del paciente (5). La educación de la población es fundamental para eliminar el estigma, realizar un diagnóstico temprano y brindar tratamiento oportunamente. Un estudio demostró que el uso de folletos educativos sobre la audición puede promover el uso de estrategias de comunicación y de servicios de salud auditiva; teniendo así un impacto en la calidad de vida de los pacientes (16). 


\section{CIENCIA\&SALUD}

Un estudio concluyó que las dietas ricas en grasas saturadas y colesterol tienen efectos perjudiciales en relación con el desarrollo de pérdida de la audición y que el daño puede prevenirse con la restricción de su consumo y el aumento de ingesta de frutas, vegetales y anti-oxidantes que tienen un efecto protector (17).

Asimismo, es de suma importancia la capacitación del personal de salud ya que los pacientes con pérdida de audición dependen de sus proveedores de salud primarios para decidir si solicitan ayuda respecto a su discapacidad. En la mayoría de casos, si el médico no pregunta, el paciente con pérdida de la audición no se siente cómodo solicitando información debido a vergüenza (18).

\section{CONCLUSIÓN}

La pérdida de audición es un problema importante en la población adulta; sin embargo, su prevención, tamizaje y tratamiento no son considerados urgencia. En muchas ocasiones, la pérdida de audición sigue siendo una discapacidad invisible, que es superada en importancia por otros problemas de salud. Durante la evaluación del paciente, es importante realizar un diagnóstico diferencial, determinar su causa e iniciar el tratamiento lo antes posible. No se debe olvidar que este padecimiento tiene gran impacto en la calidad de vida del paciente y requiere de un manejo pronto y adecuado.

\section{Financiamiento}

Autofinanciado.

\section{Conflictos de interés}

Los autores niegan tener conflictos de interés.

\section{REFERENCIAS BIBLIOGRÁFICAS}

1. Arts HA, Adams ME. Sensorineural Hearing Loss in Adults. En: Cummings. Otolaryngology. 7 ed. Philadelphia: Elsevier; 2021. p 2311-2327.

2. Swartz MH. The Ear and Nose. En: Swartz. Textbook of physical diagnosis: history and examination. 8 ed. Philadelphia: Elsevier; 2021. p 228-248.

3. Cunningham LL, Tucci DL. Hearing Loss in Adults. N Engl J Med. 2017; 377(25): 2465-2473. DOI: 10.1056/NEJMra1616601.

4. Ma Y, Wise AK, Shepherd RK, Richardson RT. New molecular therapies for the treatment of hearing loss. Pharmacol Ther. 2019; 200: 190-209. DOI:10.1016/j.pharmthera.2019.05.003.

5. Wilson BS, Tucci DL, Merson MH, ODonoghue GM. Global hearing health care: new findings and perspectives. The Lancet. 2017; 390: 2503-2515. DOI:10.1016/S01406736(17)31073-5.

6. Brown CS, Emmett SD, Kleindienst S, Tucci DL. Global Hearing Loss Prevention. Otolaryngologic Clinics of North America. 2018; 51(3): 575-592.

7. Graydon K, Waterworth C, Miller H, Gunasekera H. Global burden of hearing impairment and ear disease. J Laryngol Otol. 2019; 133: 18-25. DOI:10.1017/SO022215118001275.

8. Almeyda R, Nash R. Assessing and treating adult patients with hearing loss. British Journal of Hospital Medicine. 2018; 79(11). DOI: 10.12968/hmed.2018.79.11.628.

9. LePrell CG. Noise induced hearing loss. En: Cummings. Otolaryngology. 7 ed. Philadelphia: Elsevier; 2021. p 2342-2355.

10. Casas JS, Rodríguez D, Miranda G, De Grazia JA. Otoesclerosis: revisión de aspectos 


\section{CIENCIA\&SALUD}

etiopatogénicos, clínico-demográficos e imagenológicos. Rev Chil Rad. 2016; 22(3): 108-113. DOI: 10.1016/j.rchira.2016.08.002

11. Wackym PA. Neurotology. En: Youmans and Winn Neurological surgery. 9 ed. Philadelphia: Elsevier; 2017. p e29-e53.

12. Christopher LH, Wilkinson EP. Menieres disease: medical management, rationale for vestibular preservation and suggested protocol in medical failure. Ameriacn Journal of Otolaryngology-Head and Neck Medicine and Surgery. 2021: 42 (1). DOI: 10.1016/j.amjoto.2020.102817

13. Michels TC, Duffy MT, Rogers DJ. Hearing Loss in Adults: Differential Diagnosis and Treatment. American Family Physician. 2019; 100(2): 98-108.

14. Brodie A, Smith B, Ray J. The impact of rehabilitation on quality of life after hearing loss: a systematic review. European Archives of Oto-Rhino-Laryngology. 2018; 275: 2435-2440. DOI: 10.1007/s00405018-5100-7.

15. Sparrow K, Lind C, Steenbrugge WV. Gesture, communication and adult acquired hearing loss. Journal of Communication Disorders. 2020; 87. DOI:10.1016/j.jcomdis.2020.106030.

16. Wallhagen MI, Strawbridge WJ. Hearing loss education for older adults in primary care clinics: Benefits of a concise educational brochure. Geriatric Nursing. 2017; 38(6): 527-530. DOI: 10.1016/j. gerinurse.2017.03.015

17. Rodrigo L, Campos C, Rodríguez MA, Crespo I, Olmedillas H. Role of nutrition in the development and prevention of age related hearing loss: a scoping review. Journal of the Formosan Medical Association; 2020. DOI: 10.1016/j.jfma.2020.05.011.

18. McKee MM, Lin FR, Zazove P. State of research and program development for adults with hearing loss. Disability and Health Journal. 2018; 11(4): 519-524. DOl:10.1016/j.dhjo.2018.07.010. 